\title{
Manipulation \\ du plutonium et des transplutoniens et radioprotection $\left(^{*}\right)$
}

\author{
J. REBIFFÉ $(* *)$
}

(Manuscrit reçu le 6 avril 1976)

\begin{abstract}
RÉSUMÉ
Le plutonium est considéré comme très toxique et son seul nom engendre souvent la peur. L'article reprend d'abord les principales caractéristiques de ce corps. A partir de ces bases, des moyens de protection individuelle et collective ont été adoptés depuis de nombreuses années pour la manipulation de quantités importantes de plutonium. Les techniques de contrôle et de mesure mises au point permettent un suivi sérieux de l'état de ces moyens de prévention. Des défaillances de protection, mineures ou majeures, n'ont jamais entraîné de conséquences graves ni pour le public, ni pour les travailleurs. L'existence des moyens de protection collectifs et individuels, la qualité de la prévention et l'existence de moyens d'intervention efficaces ont contribué à l'obtention de ce résultat. Le maintien d'une prévention attentive, une collaboration de toutes les compétences, ajoutés à une expérience déjà longue permettent d'affirmer que le travail du plutonium ne présente pas plus de danger que bien d'autres activités humaines, industrielles ou non.
\end{abstract}

\begin{abstract}
Plutonium is considered as highly toxic and just its name often breeds fear. Individual and collective protection procedures in the handling of significant amounts of plutonium have been adopted for many years; they are founded on
\end{abstract}

(*) Conférence présentée lors de la Journée d'information organisée conjointement par la Société française d'Énergie nucléaire, la Section française de l'American Nuclear Society et la Société française de Radioprotection le 11 mars 1976 à l'École Centrale des Arts et Manufactures, à Châtenay-Malabry sur le thème : Toxicité et écologie du plutonium et des transplutoniens.

${ }^{(* *)}$ Commissariat à l'Énergie atomique, Centre de Valduc, B.P. $n^{\circ} 14,21220$ Is-sur-Tille.

RADIOPROTECTION, VOL. $11-\mathrm{N}^{\circ} 3$ 
its properties. Monitoring and counting rechniques make it possible to follow the conditions of these protection procedures seriously. Minor or major failures in protection have never resulted in serious consequences for the public or for workers. Such a result has been obtained thanks to the existence of collective and individual protection procedures, the quality of prevention and the availability of efficient emergency means. The keeping of careful prevention, co-operation of all proficiencies, added to a long standing experience make it possible to state that plutonium handling does not present any more danger than many other (industrial or not) human activities.

\section{INTRODUCTION}

Comment se caractérisent le plutonium et les transplutoniens pour le radioprotectioniste?

\section{TABLEAU I}

Concentrations maximales admissibles (CMA) Des PRincipaux isotopes dU PLUTONIUM

\begin{tabular}{|c|c|c|c|}
\hline $\begin{array}{c}\text { Nucléide } \\
\text { radioactif }\end{array}$ & Forme & $\begin{array}{c}\mathrm{CMA}_{\text {eau }} \\
168 \mathrm{~h} / / \mathrm{semaine} \\
\left(\mathrm{Ci} / \mathrm{m}^{3}\right)\end{array}$ & $\begin{array}{c}\mathrm{CMA}_{\text {air }} \\
168 \mathrm{~h} / \text { semaine } \\
\left(\mathrm{Ci} / \mathrm{m}^{3}\right)\end{array}$ \\
\hline \multirow{2}{*}{$238 \mathrm{Pu}$} & soluble & $5.10^{-5}$ & $7.10^{-13}$ \\
& insoluble & $3.10^{-4}$ & $1.10^{-11}$ \\
\hline \multirow{2}{*}{$239 \mathrm{Pu}$} & soluble & $5.10^{-5}$ & $6.10^{-13}$ \\
& insoluble & $3.10^{-4}$ & $1.10^{-11}$ \\
\hline \multirow{2}{*}{$240 \mathrm{Pu}$} & soluble & $5.10^{-5}$ & $6.10^{-13}$ \\
& insoluble & $3.10^{-4}$ & $1.10^{-11}$ \\
\hline \multirow{2}{*}{$241 \mathrm{Pu}$} & soluble & $2.10^{-3}$ & $3.10^{-11}$ \\
& insoluble & $1.10^{-2}$ & $1.10^{-8}$ \\
\hline \multirow{2}{*}{$242 \mathrm{Pu}$} & soluble & $5.10^{-5}$ & $6.10^{-13}$ \\
& insoluble & $3.10^{-4}$ & $1.10^{-11}$ \\
\hline \multirow{2}{*}{$243 \mathrm{Pu}$} & soluble & $3.10^{-3}$ & $6.10^{-7}$ \\
& insoluble & $3.10^{-3}$ & $6.10^{-7}$ \\
\hline \multirow{2}{*}{$244 \mathrm{Pu}$} & soluble & $4.10^{-5}$ & $1.10^{-11}$ \\
\hline
\end{tabular}

- Ils sont considérés comme très toxiques et les risques acceptés lors de leur manipulation sont très bas, d'un niveau nettement inférieur aux risques couramment admis dans l'existence.

- Certains sont émetteurs X et/ou $\gamma$ et/ou de neutrons de fission.

- Certains d'entre eux sont très fissiles.

- Ils sont, pour la plupart, pyrophoriques. 


\section{DESCRIPTION DE CES CARACTÉRISTIQUES}

\subsection{Toxicité}

Il est fondamental pour le radioprotectioniste qu'elle soit bien définie, car elle conditionne une grande part de ses attitudes et de ses décisions devant un problème de sécurité. Il est donc nécessaire que les radiobiologistes informent clairement les physiciens de santé des résultats de leurs études de radiotoxicologie. Les différentes valeurs des concentrations maximales admissibles (CMA) sont rappelées dans le tableau I.

TABLEAU II

CONCENTRATION EN ${ }^{226}$ Ra ET ${ }^{222}$ Rn DANS CERTAINES EAUX MiNÉRALES FRANÇAISES (d'après les Statistiques du Bureau de documentation minière, 1951)

\begin{tabular}{|c|c|c|c|}
\hline SOURCES & $\begin{array}{c}\text { Nature du sol } \\
\left(\mathrm{p}^{t} \text { d'émergence) }^{-}\right.\end{array}$ & $\begin{array}{c}{ }^{226} \mathrm{Ra} \\
\text { en } 10^{-6} \mu \mathrm{Ci} / \mathrm{cm}^{3}\end{array}$ & $\begin{array}{c}222 \mathrm{Rn} \\
\text { en } 10^{-6} \mu \mathrm{Ci} / \mathrm{cm}^{2}\end{array}$ \\
\hline $\begin{array}{l}\text { BOURBON } \\
\text { L'ARCHAMBAULT } \\
\text { (Allier) }\end{array}$ & $\begin{array}{l}\text { Gneiss } \\
\left(\mathrm{BiCO}_{3} \text { calco. }\right. \\
\text { sodique }) \text {. }\end{array}$ & 0,21 à 3,5 & 1,25 \\
\hline $\begin{array}{l}\text { LA PRUGNE } \\
\text { (Allier) }\end{array}$ & Granit & 12,5 & \\
\hline $\begin{array}{l}\text { SAINT-YORRE } \\
\text { PARMENTIER } \\
\text { (Allier) }\end{array}$ & $\begin{array}{l}\text { Sédim. Tertiaire } \\
\left(\mathrm{CO}_{3} \mathrm{HNa}\right)\end{array}$ & 6,24 & 18,2 \\
\hline $\begin{array}{l}\text { AX-les-THERMES } \\
\text { (Ariège) }\end{array}$ & $\begin{array}{l}\text { Pegmatite-schistes } \\
\text { (sulfureuse sodi- } \\
\text { que) }\end{array}$ & 3,39 & 40,9 \\
\hline $\begin{array}{l}\text { CHATELDON } \\
\text { (Puy-de-Dôme) }\end{array}$ & $\begin{array}{l}\text { Granit } \\
\left(\mathrm{CO}_{3} \mathrm{HNa}\right)\end{array}$ & 130 & \\
\hline $\begin{array}{l}\text { MAGNIEN } \\
\text { (Côte d'Or) }\end{array}$ & $\begin{array}{l}\text { Porphyre } \\
\text { (chl. sod.) }\end{array}$ & 10,57 & 45 à 71 \\
\hline $\begin{array}{l}\text { VERNET-LES-BAINS } \\
\text { (Pyrénées-Orientales) }\end{array}$ & Gneiss (sulf. sod.) & 15,7 & 115,9 \\
\hline $\begin{array}{l}\text { BAGNERES DE LUCHON } \\
\text { (Haute-Garonne) }\end{array}$ & $\begin{array}{l}\text { Granit } \\
\text { (sulf. sodique) }\end{array}$ & 6,3 à 26,2 & 90 à 204 \\
\hline $\begin{array}{l}\text { Maximum }\left\{\begin{array}{l}{ }^{226} \mathrm{Ra}= \\
{ }^{222} \mathrm{Rn}=\end{array}\right. \\
\text { En comparaison CMA }={ }^{22}\end{array}$ & $\begin{array}{l}1.3 \cdot 10^{-4} \mu \mathrm{Ci} / \mathrm{cm}^{3} \\
2.10^{-4} \mu \mathrm{Ci} / \mathrm{cm}^{3} \\
=4 \cdot 10^{-9} \mu \mathrm{Ci} / \mathrm{cm}^{3}\end{array}$ & \multicolumn{2}{|c|}{$\begin{array}{l}\text { (CHATELDON) } \\
\text { (BAGNERES-DE-LUCHON) }\end{array}$} \\
\hline
\end{tabular}

vol. $11-\mathrm{N}^{\circ} 3$ 


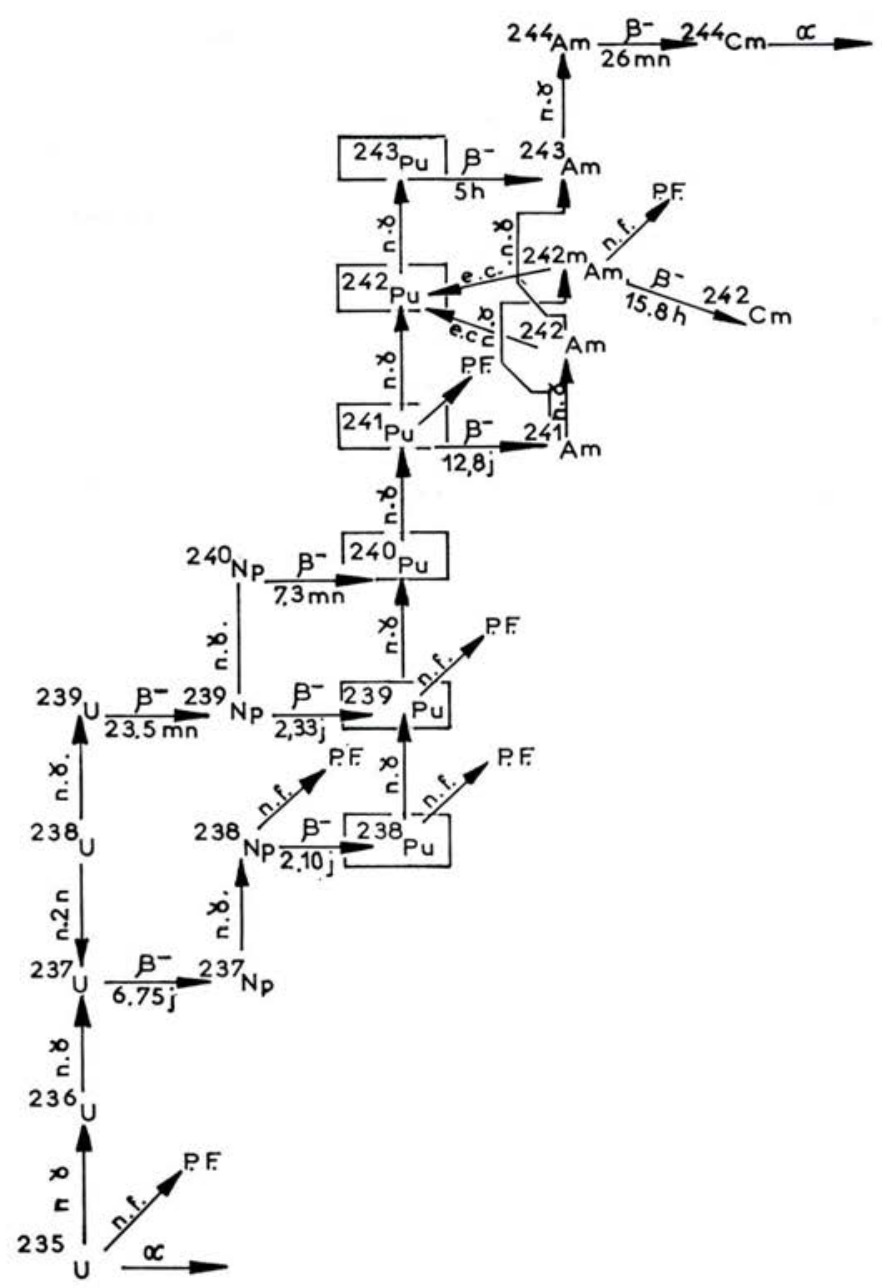

FIG. 1. - Famille du plutonium.

On constate que si cette toxicité n'est pas négligeable, celle du radium n'en est pas éloignée, en particulier en ce qui concerne les eaux de boisson. On rapprochera avec intérêt ces valeurs de celles rencontrées dans certaines eaux minérales françaises (tableau II).

\subsection{La famille du Plutonium. IrRadiation}

Sans décrire dans le détail l'ensemble des caractéristiques de cette famille, on remarque ( $f i g .1$, tableau III) :

- des pourcentages importants d'émission X; 
TABLEAU III

CARACTÉRISTIQUes de Certains ISOTOPES dU PLUTONIUM ET DE L'AMÉRICIUM

\begin{tabular}{|c|c|c|c|c|c|c|}
\hline \multirow{2}{*}{ Isotope } & \multirow{2}{*}{ Période } & \multicolumn{3}{|c|}{ Rayonnement } & \multirow{2}{*}{$\begin{array}{c}\text { Fissions } \\
\text { spontanées }\end{array}$} & \multirow{2}{*}{$\begin{array}{l}\text { Mode de } \\
\text { formation }\end{array}$} \\
\hline & & nature & Energie en $\mathrm{MeV}$ & Pourcentage & & \\
\hline \multirow{3}{*}{237} & \multirow{3}{*}{$\begin{array}{c}45,63 \pm 0,20 \\
\text { jours }\end{array}$} & $\alpha$ & $\begin{array}{l}5,66 \\
5,37\end{array}$ & $\begin{array}{l}21 \text { (rela.) } \\
79 \text { (rela.) }\end{array}$ & & $\begin{array}{l}{ }^{235} \mathrm{U}(\alpha, 2 n) \\
{ }^{238} \mathrm{U}(\alpha, 5 n)\end{array}$ \\
\hline & & $\gamma$ & 0,060 & 5 & & ${ }^{237} \mathrm{~Np}(\mathrm{~d}, 2 \mathrm{n})$ \\
\hline & & $\mathrm{e}^{-}$ & $\begin{array}{l}0,026-0,032- \\
0,038-0,042- \\
0,056\end{array}$ & & & \\
\hline \multirow{4}{*}{238} & \multirow{4}{*}{86,0 ans } & $\alpha$ & $\begin{array}{l}5,499 \\
5,456\end{array}$ & $\begin{array}{l}72 \\
28\end{array}$ & \multirow{4}{*}{$\begin{array}{l}\text { Période : } \\
4,9.10^{10} \text { ans } \\
\\
\text { neutrons/s/g } \\
3,44.10^{3} \\
\\
\text { neutrons par } \\
\text { fission : } 2,33\end{array}$} & \multirow{4}{*}{$\begin{array}{l}{ }^{237} \mathrm{~Np}(\mathrm{n}, \gamma) \\
{ }^{238} \mathrm{~Np} \stackrel{\beta}{\rightarrow} \\
{ }^{238} \mathrm{U}(\alpha, 4 \mathrm{n}) \\
{ }^{235} \mathrm{U}(\alpha, \mathrm{n}) \\
{ }^{242} \mathrm{Cm} \rightarrow{ }^{\alpha} \\
{ }^{241} \mathrm{Am}(\mathrm{n}, \alpha)\end{array}$} \\
\hline & & $\gamma$ & $\begin{array}{l}0,099 \\
0,150\end{array}$ & $\begin{array}{r}8.10^{-3} \\
10^{-3}\end{array}$ & & \\
\hline & & $\mathrm{e}^{-}$ & $0,024-0,039$ & & & \\
\hline & & $\mathrm{X}$ & 0,017 & 13 & & \\
\hline \multirow{4}{*}{239} & \multirow{4}{*}{$\begin{array}{c}24386 \\
\pm 26 \text { ans }\end{array}$} & $\alpha$ & $\begin{array}{l}5,157 \\
5,145 \\
5,107\end{array}$ & $\begin{array}{l}73,3 \\
15,1 \\
11,5\end{array}$ & \multirow{4}{*}{$\begin{array}{l}\text { Période : } \\
5,5.10^{15} \text { ans } \\
\text { neutrons/s/g: } \\
0,03 \\
\text { neutrons par } \\
\text { fission } \\
\sim 2,14\end{array}$} & \multirow{4}{*}{$\begin{array}{l}{ }^{238} \mathrm{U}(\alpha, 3 \mathrm{n}) \\
{ }^{239} \mathrm{~Np} \stackrel{\beta}{\rightarrow} \\
{ }^{238} \mathrm{Pu}(\mathrm{n}, \gamma)\end{array}$} \\
\hline & & $\gamma$ & $\begin{array}{l}0,039 \\
0,052 \\
0,129 \\
0,375 \\
0,414 \\
0,65\end{array}$ & $\begin{array}{c}7.10^{-3} \\
2.10^{-3} \\
5.10^{-3} \\
1,2.10^{-3} \\
1,2.10^{-3} \\
8.10^{-5}\end{array}$ & & \\
\hline & & $\mathrm{e}^{-}$ & $\begin{array}{l}0,008-0,019- \\
0,033-0,047\end{array}$ & & & \\
\hline & & $\mathbf{X}$ & $\begin{array}{l}0,0136 \\
0,0174 \\
0,0205\end{array}$ & $\begin{array}{l}1,4 \\
2,2 \\
0,2\end{array}$ & & \\
\hline \multirow{4}{*}{241} & \multirow{4}{*}{$434 \pm 19$ ans } & $\alpha$ & $\begin{array}{l}5,486 \\
5,443 \\
5,389 \\
\end{array}$ & $\begin{array}{l}86 \\
12,7 \\
1,3 \\
\end{array}$ & \multirow{4}{*}{$\begin{array}{l}\text { Période : } \\
2,3.10^{14} \text { ans } \\
\text { Neutrons/s/g } \\
0,623 \\
\text { Neutrons par } \\
\text { fission } \\
\sim 2,16\end{array}$} & \multirow{4}{*}{${ }^{241} \mathrm{Pu} \stackrel{\beta-}{\rightarrow}$} \\
\hline & & $\gamma$ & $\begin{array}{l}0,060 \\
0,026 \\
0,033\end{array}$ & $\begin{array}{l}40 \\
2,8 \\
0,08\end{array}$ & & \\
\hline & & $\mathrm{e}^{-}$ & $\begin{array}{l}0,022-0,038- \\
0,054\end{array}$ & & & \\
\hline & & $\mathbf{X}$ & 0,017 & $\sim 35$ & & \\
\hline \multirow{4}{*}{242} & \multirow{4}{*}{$16,01 \mathrm{~h}$} & $\beta^{-}$ & $0,67 \max$ & 84 & & \multirow{4}{*}{$\begin{array}{l}{ }^{241} \mathrm{Am}(\mathrm{n}, \gamma) \\
{ }^{242} \mathrm{Am} \stackrel{\gamma-}{\rightarrow}\end{array}$} \\
\hline & & C.E. & & 16 & & \\
\hline & & $\mathrm{e}^{-}$ & $0,021-0,037$ & & & \\
\hline & & $\gamma$ & $\begin{array}{l}0,042 \\
0,044\end{array}$ & & & \\
\hline
\end{tabular}


- des fissions spontanées amenant une émission neutronique;

- la croissance dans le temps de ${ }^{241} \mathrm{Am}$ fils de ${ }^{241} \mathrm{Pu}$.

Dans la pratique viennent s'ajouter :

- l'existence de traces de produits de fission, même après traitement;

- le fait que l'on a presque toujours affaire à des mélanges isotopiques.

Ces deux derniers points prennent une importance d'autant plus grande que le taux de combustion en pile est plus élevé.

Exemple :

- Pu graphite gaz $\sim 4 \mathrm{rad} . \mathrm{h}^{-1}$ au contact d'une source épaisse de $\mathrm{PuO}_{2}$ $\sim 0,050 \mathrm{rad} . \mathrm{h}^{-1}$ avec $4 \mathrm{~mm}$ de plomb de protection.

- Pu eau légère $\sim 16 \mathrm{rad} \cdot \mathrm{h}^{-1}$ au contact d'une source épaisse de $\mathrm{PuO}_{2}$ $\sim 0,100 \mathrm{rad} \cdot \mathrm{h}^{-1}$ avec $4 \mathrm{~mm}$ de plomb de protection.

Ceci pour les raies de 17 à $60 \mathrm{keV}$ du Plutonium.

Dans ces conditions, il est difficile de prévoir avec une grande rigueur les niveaux d'irradiation probables aux postes de travail. La formule suivante de NoEsh et FAust permet une estimation du débit de dose pour les composantes $\mathrm{X}$ et $\gamma$ du rayonnement émis :

$$
\begin{aligned}
D_{s}= & 960 F_{238}+0,98 F_{239}+4,7 F_{240} \\
& +\left[0,19 T+17\left(1-e^{-0,102 T}\right)\right] F_{241}+0,29 F_{242}
\end{aligned}
$$

$D_{s}$, irradiation de surface en rad. $\mathrm{h}^{-1}$;

$F_{i}$, pourcentage en poids de l'isotope;

$T$, temps écoulé depuis la séparation, en jours.

A cette émission $X$ et $\gamma$ peut venir s'ajouter l'irradiation par les neutrons de fission ou provenant de réactions $(\alpha, n)$ émission qui peut augmenter nettement le débit de dose. Le facteur " forme de la pièce " modifie également le débit de dose et est à prendre en compte. Enfin, en pratique, on constate que les poussières accumulées dans les enceintes de travail (boîtes à gants, par exemple) peuvent conduire à des niveaux d'irradiation équivalents à ceux donnés par les pièces elles-mêmes. Un nettoyage régulier de ces enceintes est donc indispensable.

En conclusion, on constate que l'approche de la connaissance des débits de dose prévisionnels, par extrapolation à partir des mesures faites sur des postes de travail existants, est certainement la méthode la plus féconde et celle qui amène le moins d'erreur dans la définition des protections à adopter.

\subsection{CRITICITÉ}

- Certains des isotopes considérés sont fissiles. C'est le cas du plutonium 239.

Ce caractère fissile peut conduire à des accidents dits de criticité. Ces accidents sont caractérisés par une très forte émission de rayonnements 


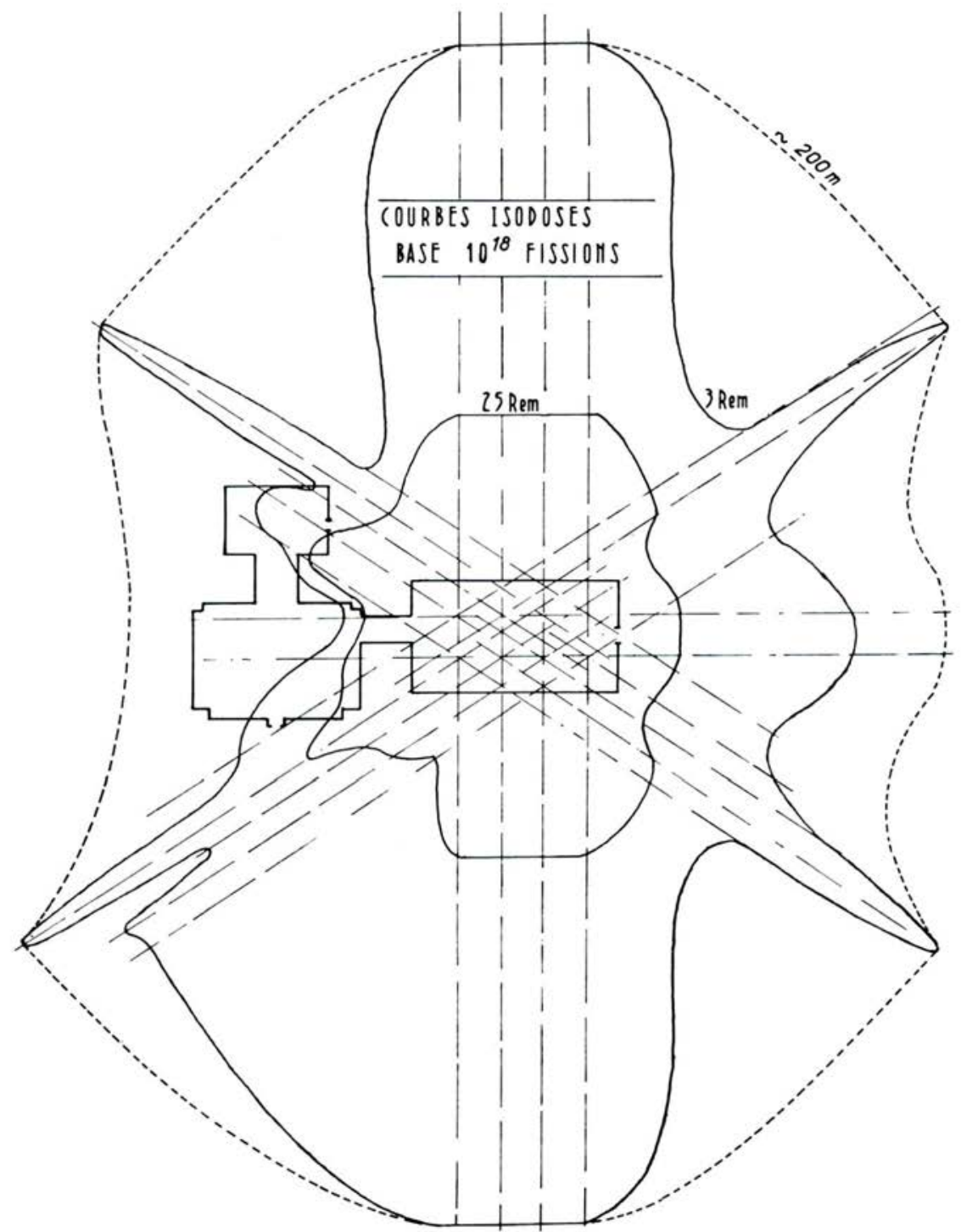

FIG. 2. - Courbes isodoses en cas d'accident de criticité (exemple).

accompagnant les quelque $10^{18}$ fissions produites et, en conséquence, par des débits de dose très élevés dans les quelques mètres situés autour du point d'accident ( fig. 2).

Il convient de tout mettre en œuvre pour éviter de tels accidents. Les études menées dans ce domaine, en France, à la station de Criticité de Valduc,

voL. $11-\mathrm{N}^{\circ} 3$ 
ainsi qu'au Service d'Études Critiques de Saclay, assurent des pronostics extrêmement sûrs et permettent, dans des conditions définies, des manipulations de très grandes quantités de matières fissiles, compatibles avec les programmes nucléaires envisagés.

\subsection{Pyrophoricité. OXYDABILITÉ}

Le Plutonium métal et certains autres transuraniens sont pyrophoriques.

Ce caractère implique des techniques particulières de prévention contre l'incendie et de protection contre la corrosion (travail sous atmosphère inerte, par exemple) ( $c f$. $§ 2$. Prévention).

\section{PRÉVENTION}

Compte tenu de ces caractéristiques, comment depuis vingt ans a-t-on imaginé la prévention dans la manipulation de ces éléments en France?

\subsection{Prévention Collective}

\subsubsection{Installations générales}

Le principe adopté pour la réalisation des installations dans lesquelles on travaille le plutonium est le confinement, ceci de façon à exclure toute dispersion des toxiques dans l'environnement. Ce confinement est réalisé à l'aide de ventilations puissantes, de filtres à haut pouvoir d'arrêt créant un gradient de dépression croissant de l'extérieur vers l'intérieur des locaux. Ces locaux sont aménagés en zones ( fig. 3).

Le confinement extrême est assuré au niveau d'enceintes dites boîtes à gants également en dépression (-10 à $20 \mathrm{~mm}$ de CE (colonne d'eau) par rapport au local dans lequel elles sont implantées) possédant également un système de filtration indépendant. Ces enceintes peuvent recevoir, si nécessaire, des panneaux de verre au plomb (protection X et $\gamma$ ) et/ou des protections neutroniques. Il existe des boîtes à gants de toutes dimensions. Certaines sont capables de recevoir les plus grandes machines-outils actuelles. Ce travail en boîtes à gants individuelles, quelles que soient leurs dimensions, reste artisanal ou, à la rigueur, semi-industriel.

Les quantités à manipuler dans l'avenir impliquent des méthodes de travail et de transfert plus automatisées. Ces procédés introduisent, si l'on n'y prend pas garde, des risques de propagation d'incendie par les systèmes automatiques de transfert. Des analyses attentives doivent être réalisées lors de l'étude des postes de travail.

Toutes ces installations font l'objet d'études de sûreté approfondies qui s'appuient, entre autres, sur la théorie dite des barrières, très adaptée aux études de sûreté des réacteurs. On s'assure, à l'analyse, qu'aucune solution de continuité n'existe ou ne peut être créée accidentellement dans ces barrières (cloisons, système de filtration, boîtes à gants, circulation du personnel, etc.) et on examine les conséquences d'éventuelles ruptures. 


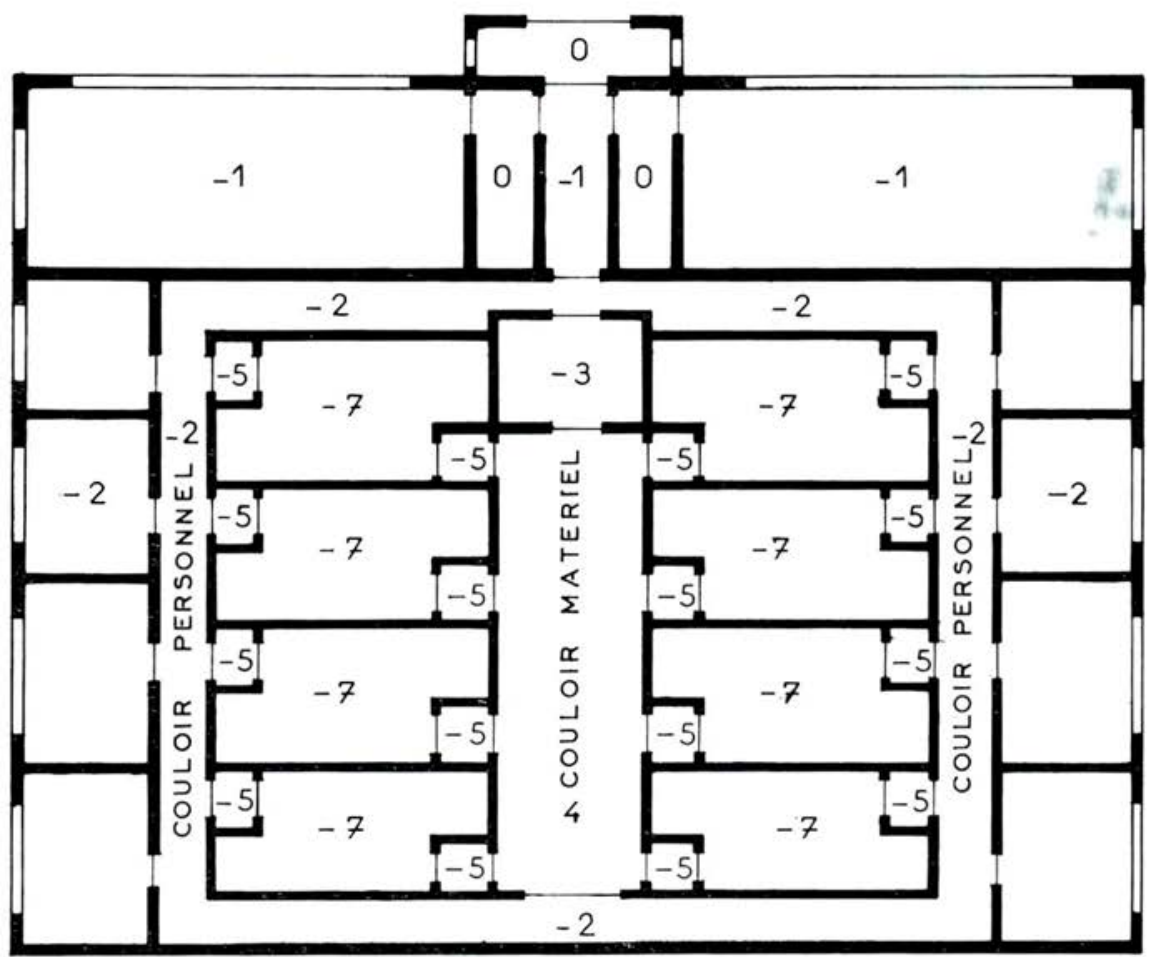

SCHEMA D'UN BATIMENT

Fig. 3. - Schéma d'un laboratoire de travail du plutonium.

La Commission de Sûreté des Laboratoires et Usines est chargée, au C.E.A., de l'étude des dossiers de sûreté de ces installations.

\subsubsection{Téléalarme}

L'existence d'un excellent réseau de téléalarme contribue pour beaucoup à la réalisation d'une bonne prévention collective. Ce réseau doit permettre, entre autres :

- les demandes de secours gradués,

- la transmission d'informations de sûreté (mesures radioactives, informations techniques concourant à la sûreté, dépression, boîte à gants, par exemple).

\subsubsection{Incendie}

La prévention contre le feu est capitale. L'incendie est certainement le vecteur principal de la dispersion possible des produits manipulés. Les grands

vOL. $11-\mathrm{N}^{\circ} 3$ 
accidents survenus dans le monde, dans ce domaine, ont tous cette cause pour origine. C'est à partir de l'avis de spécialistes compétents qu'est bâtie la sûreté incendie. Ces spécialistes peuvent guider le constructeur sur le choix des meilleurs systèmes de détection et des matériaux les plus adaptés. Mais la prévention passe également par la vérification périodique ( 2 fois par an) du bon état de santé des bâtiments et des équipements sous l'angle de l'incendie.

\subsubsection{Criticité}

La prévention collective est d'abord assurée, en ce qui concerne la criticité, par l'étude très fine des procédés. Un dossier de sûreté doit être constitué, constamment tenu à jour et soumis au jugement des experts (Commission de Sûreté Criticité au C.E.A.). Une modification qui peut paraître mineure dans un procédé peut avoir une incidence sérieuse sur la sûreté criticité (changement de dimensions d'un moule à la fusion, stockage modifié, vanne changée de position, etc.). La plus grande rigueur est indispensable.

Si ces conditions sont respectées, on peut alors travailler en toute sécurité sur des quantités importantes.

Les figures 4 à 7 mettent en évidence les précautions prises pour satisfaire cette sûreté lors de transferts de plutonium métal ou lors de stockage (respect de géométrie, masses définies, concentrations définies).

\subsubsection{Effluents}

Aucun rejet d'effluent liquide suspect ne peut se faire sans passer par l'intermédiaire de capacités de rétention permettant les contrôles, aussi bien des corps radioactifs que des toxiques chimiques éventuellement contenus dans les liquides à rejeter.

\subsection{6. Énergie secourue}

Enfin, la protection collective est effective et complète si un réseau d'énergie secourue permet de pallier les défaillances possibles du réseau E.D.F. et leurs conséquences sur l'installation (alimentation des opérations en cours, téléalarme, moyens de mesure de radioprotection, etc.).

\section{Exemple :}

Coût d'un bâtiment dans lequel on manipule plusieurs dizaines de kilogrammes de plutonium par an :
- gros-œuvre :
50 p. cent;
- fluides divers :
11 p. cent;
- sécurité-ventilation : 20 p. cent (ventilation 15 p. cent);
- électricité : 13 p. cent;

pour un coût total de 50 MF (actualisés). 


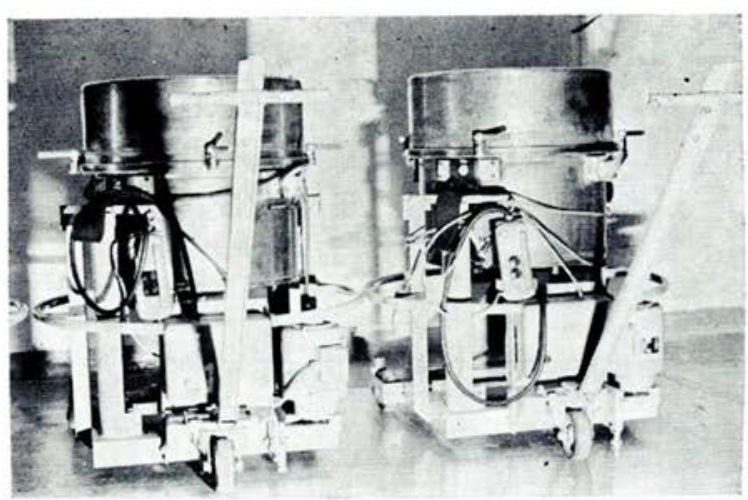

Fig. 4

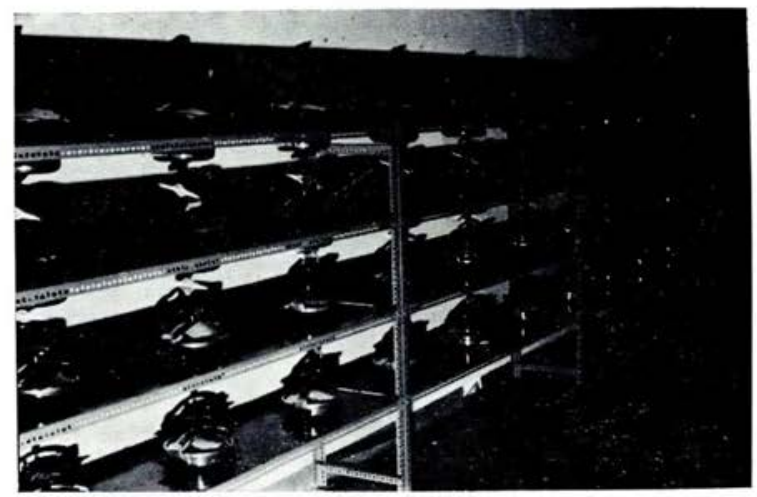

Fig. 5

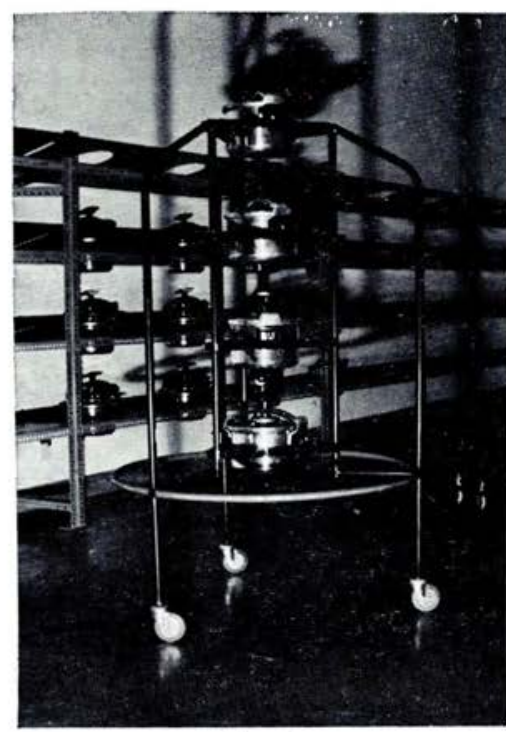

Fig. 6

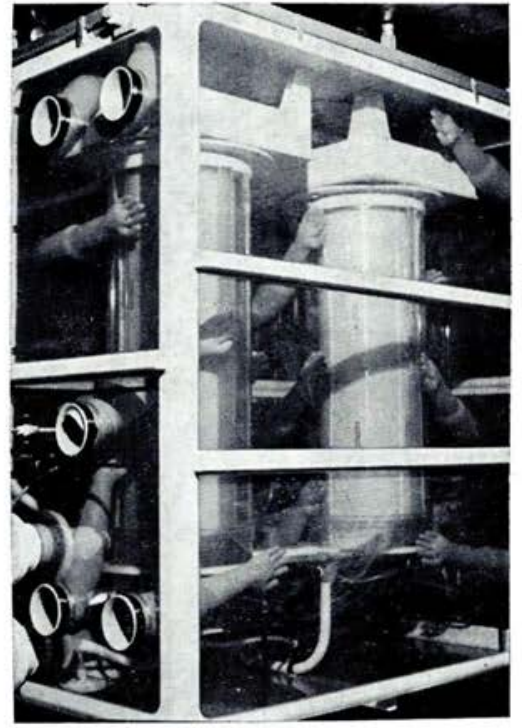

Fig. 7

FIG. 4 à 7. - Stockage et transfert de matières fissiles. 


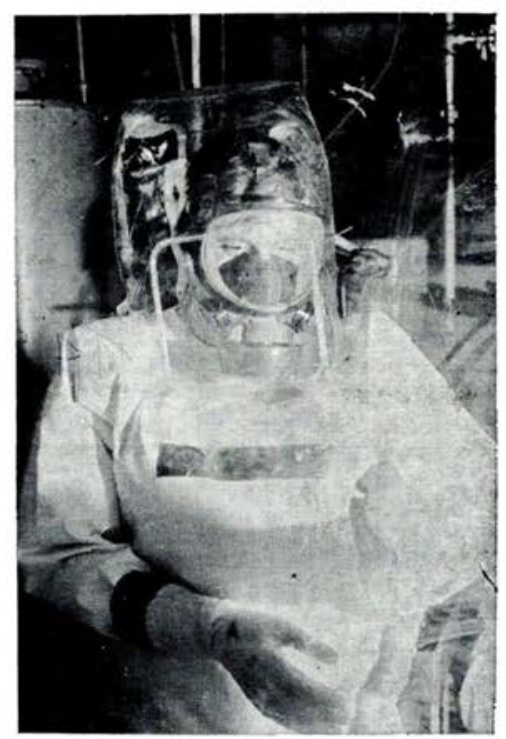

Fig. 8

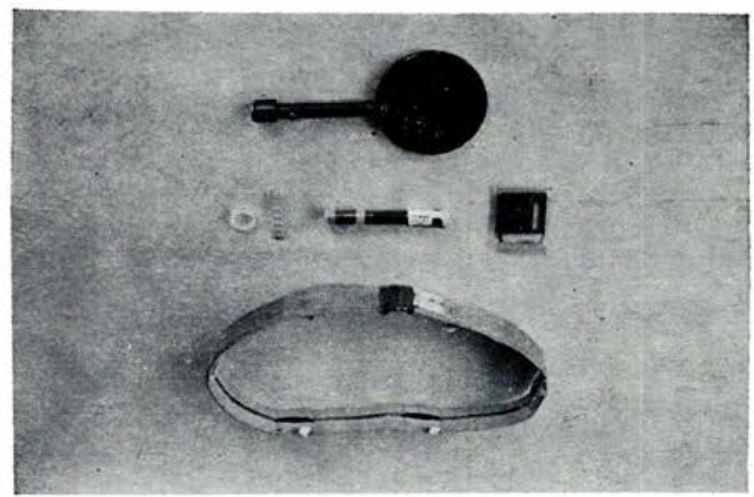

Fig. 9

FIG. 8, 9. - Protection individuelle.

\subsection{PRÉVENTION INDIVIDUelle}

Elle passe par la rédaction de consignes de sûreté simples et compréhensibles par tous, largement diffusées et commentées, remises régulièrement à jour. Une technique efficace consiste à extraire de consignes générales l'essentiel des mesures de sécurité, afin de ne pas noyer l'important dans le détail. S'ajoutent, bien entendu, à ces consignes, des moyens individuels de protection : masques adaptés, tenues particulières, scaphandres, etc. ( $f i g .8$ et 9). 


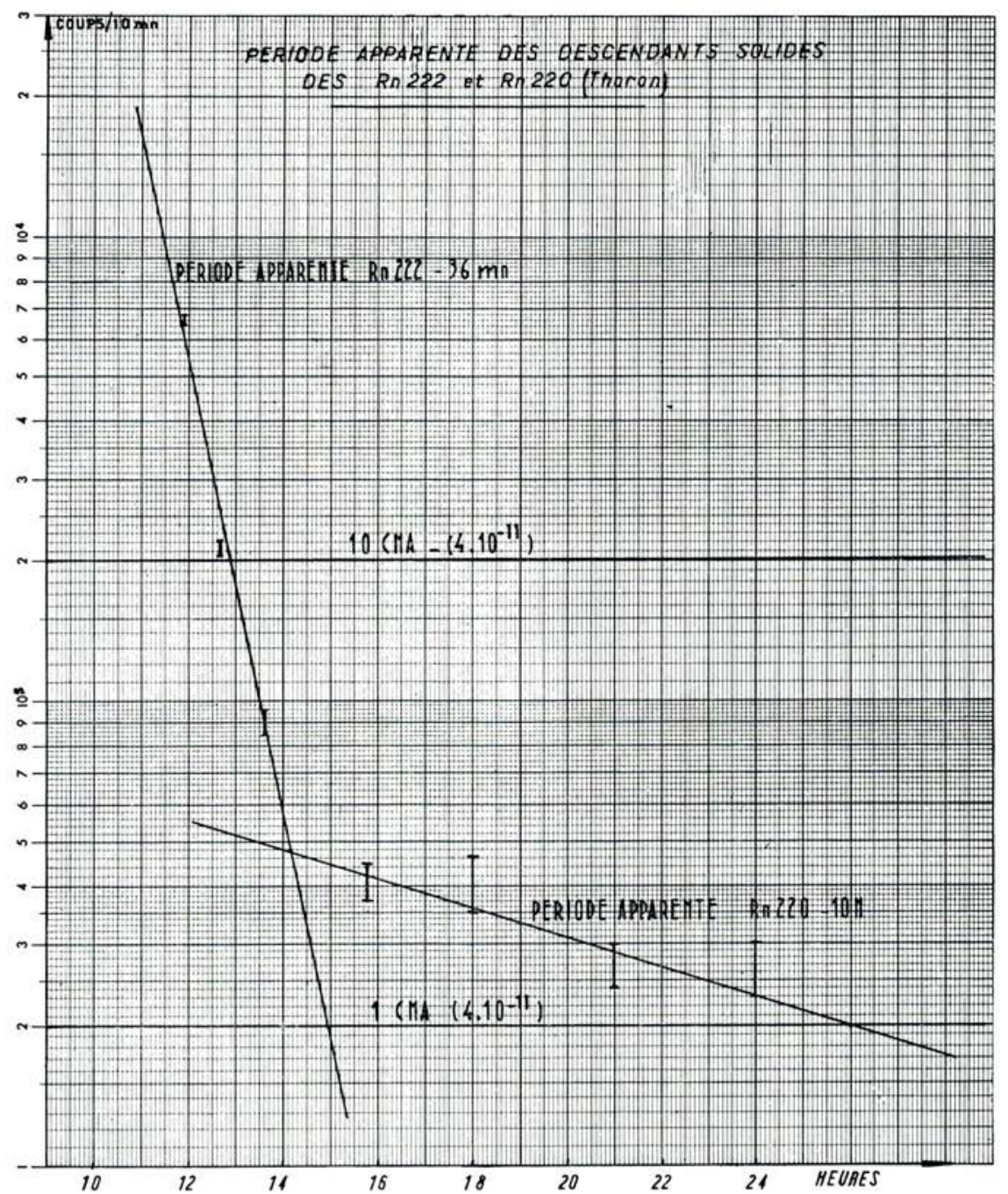

FIG. 10. - Période apparente des descendants solides des ${ }^{222} \mathrm{Rn}$ et ${ }^{220} \mathrm{Rn}$ (thoron). (La CMA portée sur le graphique est la CMA 40h - Pu insoluble)

\section{CONTRÔLES ET MESURES}

Les contrôles et mesures du plutonium et des transuraniens se caractérisent par :

- un échantillonage difficile,

voL. $11-\mathrm{N}^{\circ} 3$ 
- l'existence d'un bruit de fond constitué par les dépôts solides du radon et du thoron;

- des difficultés de mesure de la contamination des surfaces.

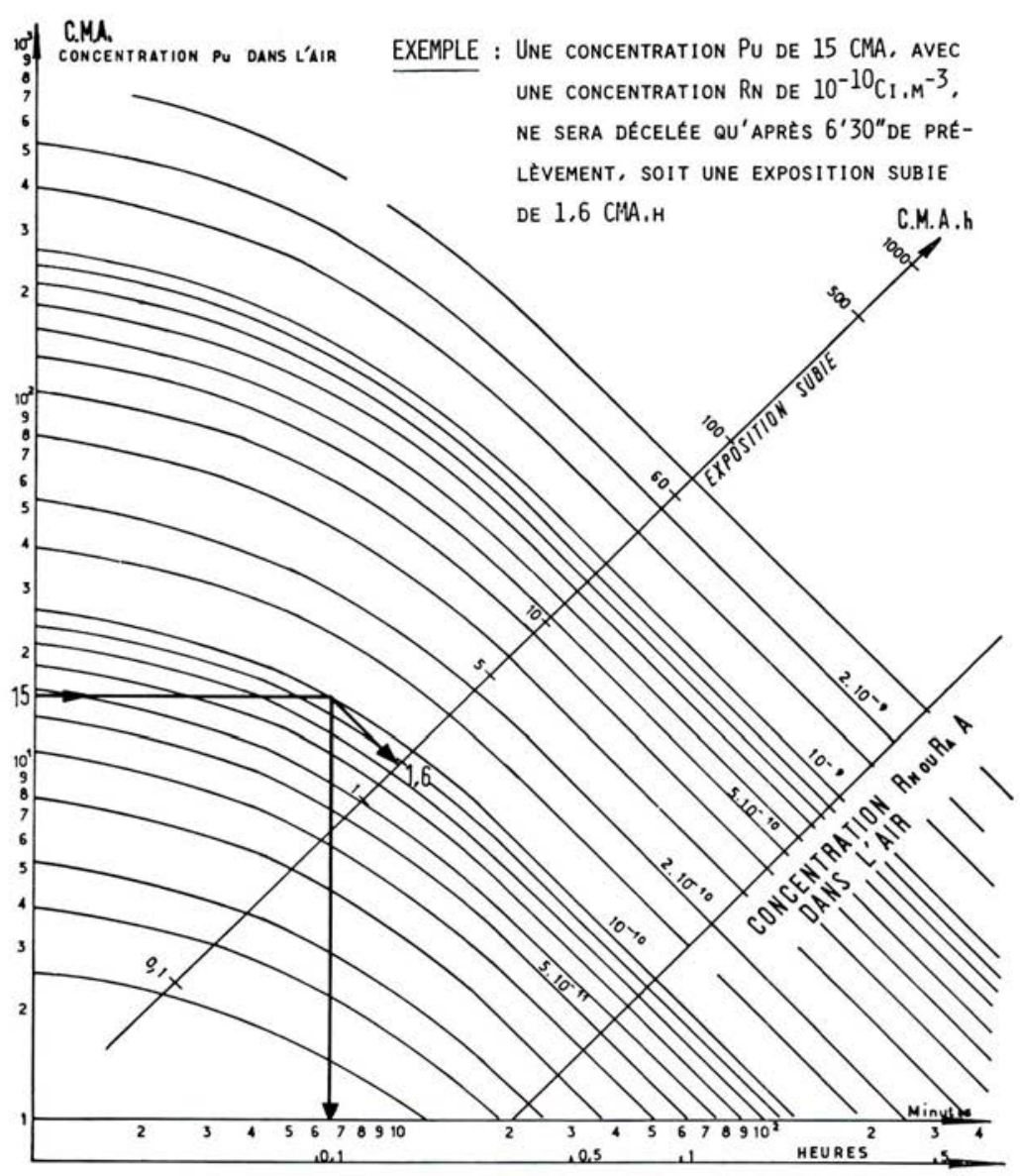

FIG. 11. - Détection du plutonium par spectrométrie alpha - caractéristiques.

\subsection{CONTRÔLES ATMOSPhÉRIQUES}

A titre d'illustration, un calcul rapide montre que la concentration maximale admissible dans $1 \mathrm{~m}^{3}$ est obtenue avec une poussière de $1,5 \mu \mathrm{m}$ de diamètre. Or, on considère qu'il faut environ $10^{6}$ particules par mètre cube pour qu'un échantillonnage soit valable. Ce qui met bien en évidence la difficulté d'obtenir des prélèvements parfaitement représentatifs. 
La courbe jointe ( fig. 10) met en évidence la deuxième difficulté liée à l'existence des dépôts solides du radon et du thoron - dans les premières heures qui suivent un prélèvement. Ces difficultés sont connues et prises en

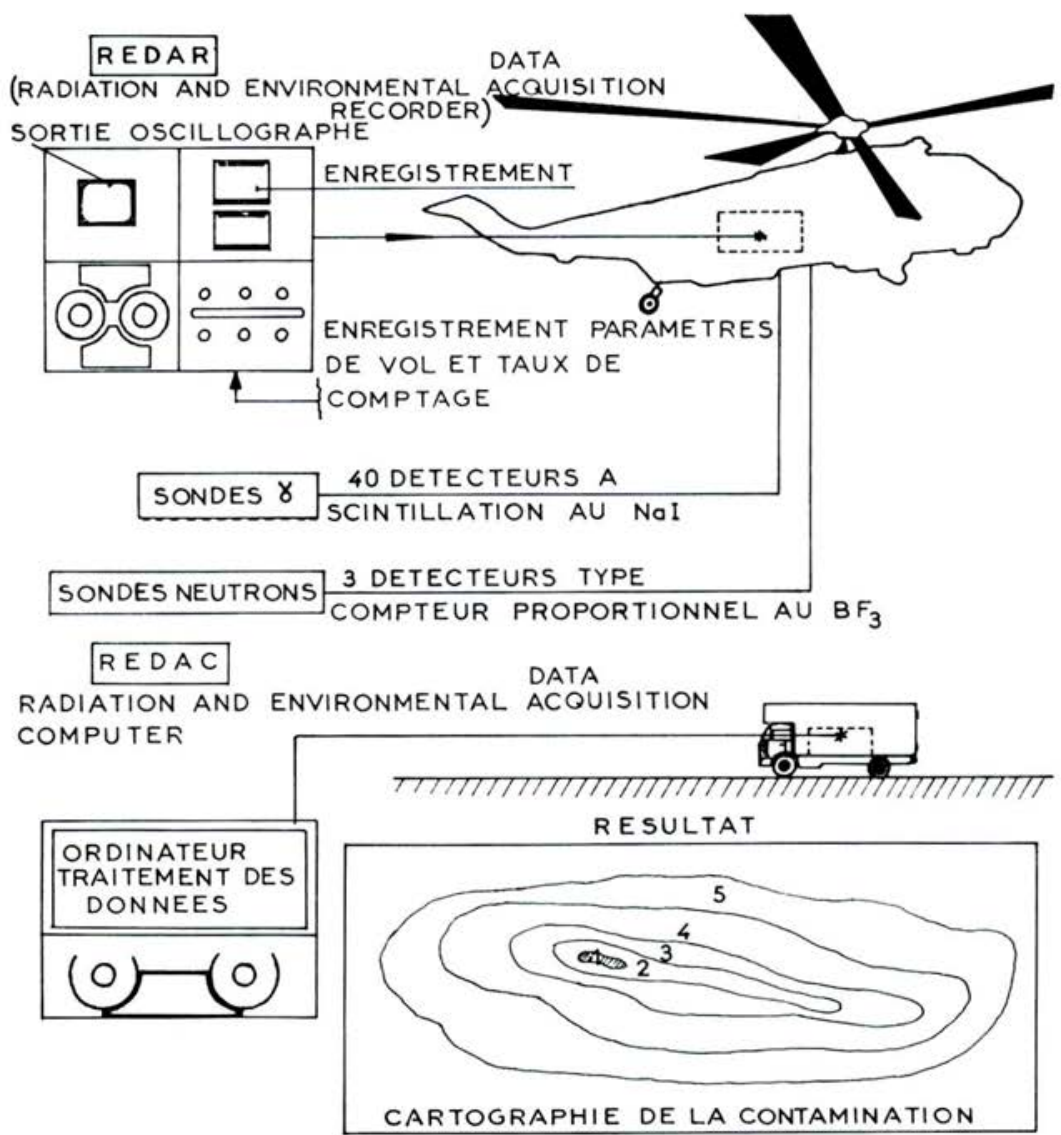

Fig. 12. - Système de détection par voie aérienne en $\gamma$ et en neutrons - schéma général.

compte dans la conception des équipements de mesure, depuis l'origine du travail sur plutonium (utilisation des coïncidences $\alpha, \beta$, inertie différentielle des poussières, etc.).

Bien entendu, les performances du matériel se sont améliorées et les moyens utilisés actuellement permettent une bonne détection, c'est-à-dire rapide, sensible et sélective, des corps à longue période. Il n'est pas possible de citer l'ensemble du matériel utilisé pour la détection, dans le cadre de cet article. A titre d'exemple est donnée (fig. 11) la courbe de réponse d'un

VOL. $11-\mathrm{N}^{0} 3$ 
appareil de contrôle de contamination atmosphérique qui utilise la spectrographie $\alpha$ par diode sur un canal. L'échantillonnage n'est pas réglé d'une façon aussi satisfaisante mais, dans ce domaine, chaque laboratoire représente un cas particulier.

\subsection{Contrôles DE SURFACE}

L'évolution dans le temps a suivi le progrès de l'électronique et le matériel est devenu plus compact et plus fiable. A part les contrôles utilisant l'émission $\alpha$

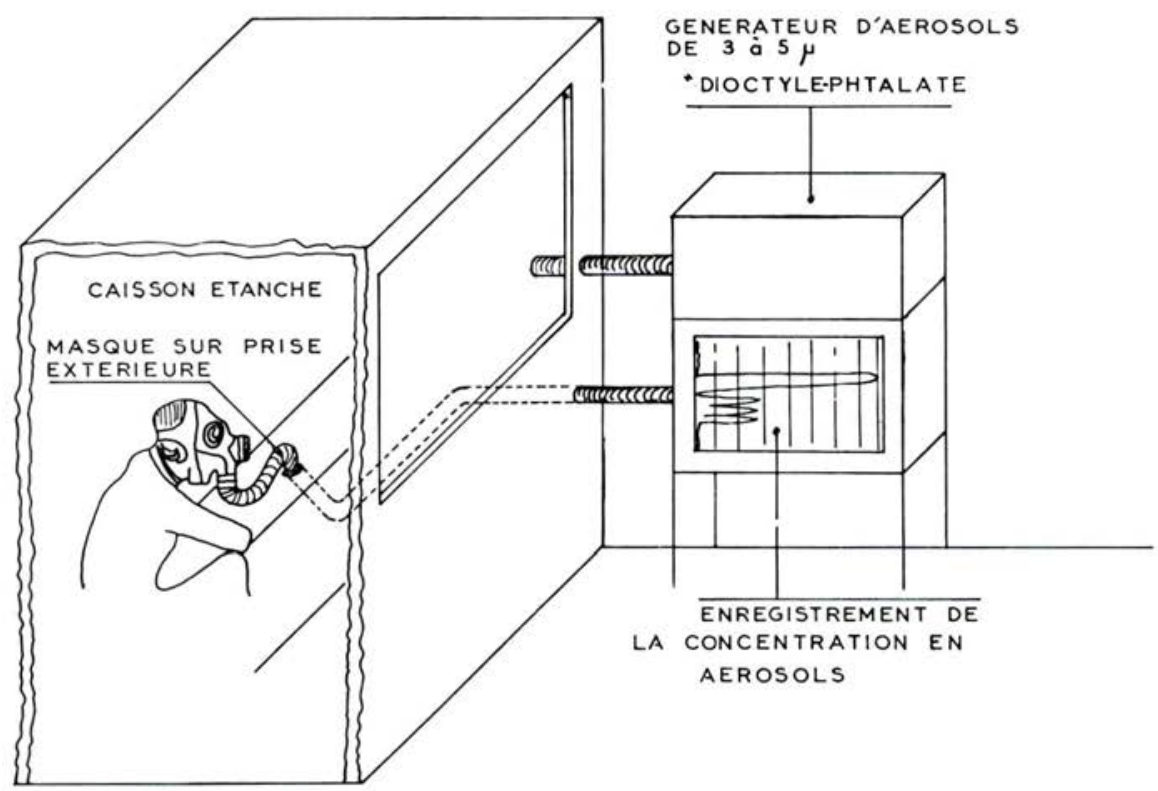

FIG. 13. - Contrôle d'étanchéité des masques et des scaphandres - principe.

du plutonium ou des transuraniens, il convient de citer une évolution récente utilisant les raies $\mathrm{X}$, qui facilite considérablement la détection dans certains cas particuliers (accidents importants, par exemple). Cette technique extrapolée peut être utilisée par voie aérienne dans de nombreux cas. C'est le but d'études appliquées, en cours sur le Centre de Valduc : accidents d'armes, surveillance périodique des sites nucléaires (évolution de l'impact sur l'environnement), recherche de sources perdues, météorologie, etc. (fig. 12).

\subsection{Mesures individuelles}

Citons, sans les décrire : films dosimètres, stylos dosimètres, ceintures de criticité, dosimètres bagues LiF ( fig. 9), mesures de la radioactivité du mucus nasal, mesures radiotoxicologiques. 


\subsection{Côntroles et MESURES Diverses}

A tous ces contrôles spécifiques qui tissent une trame serrée, s'ajoute et les complète efficacement, une série de contrôles divers dont l'existence est précieuse, même si tous n'ont pas, pour composante principale, la sécurité.

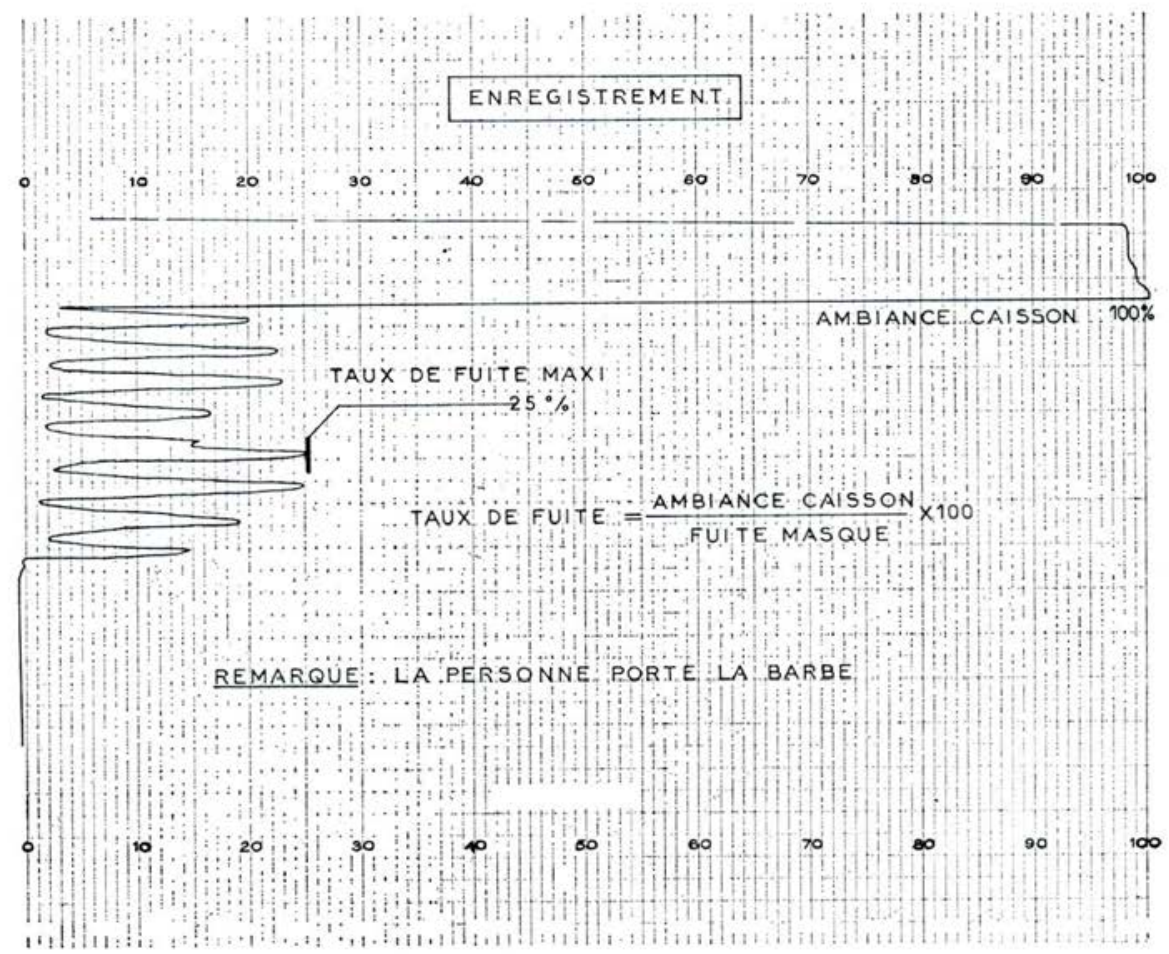

FIG. 14. - Courbe de fuite d'un masque (due au port de la barbe).

- surveillance et contrôle des masques et des scaphandres, réception et contrôle des gants des boîtes à gants, ventilation, effluents des circuits secondaires de refroidissement, hottes et sorbonnes, chaînes de criticité, réseau de téléalarme, véhicules de transport des matières radioactives, sources scellées, tenues de travail, etc.

A titre d'exemple sont donnés en schéma dans les figures 13 et 14 :

- Le principe de contrôle des masques et des scaphandres.

- Une courbe mettant en évidence la mauvaise étanchéité d'un masque, due au port de la barbe.

VOL. $11-\mathrm{N}^{\circ} 3$ 
J. REBIFFÉ

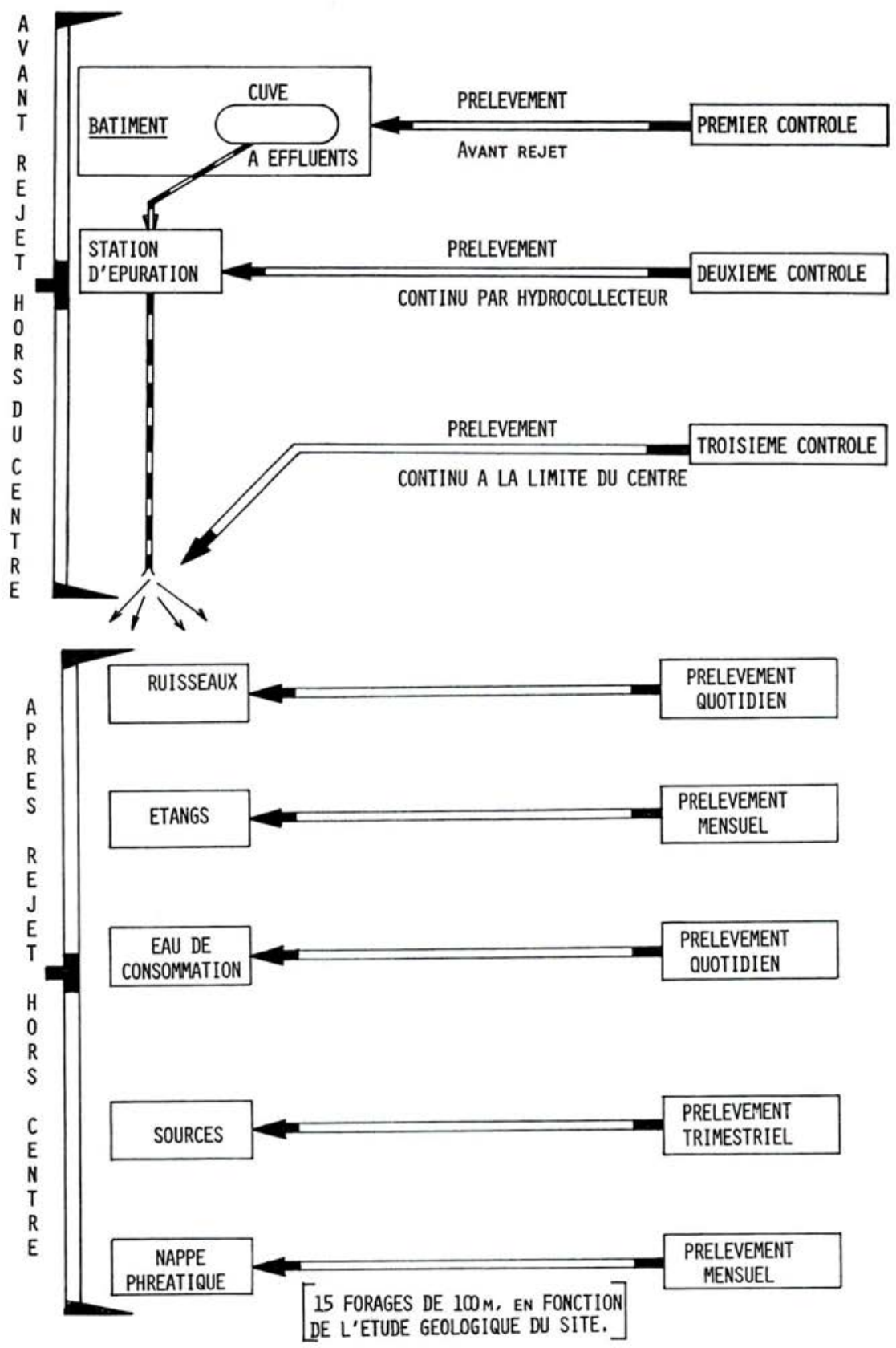

FIG. 15. - Schéma de la surveillance d'un site. 


\subsection{Surveillance du Site}

Elle est l'enveloppe de tous les autres contrôles ( fig. 15).

\section{INCIDENTS. ACCIDENTS. INTERVENTION}

Les incidents ou accidents ont, la plupart du temps, comme origine une rupture de confinement. Les principales ruptures de confinement mineures apparaissent dans le tableau ci-dessous :

\begin{tabular}{|c|c|}
\hline Types d'incidents les plus fréquents & p. cent \\
\hline Gants ou sacs d'introduction percés en cours de manipulation $\ldots \ldots \ldots \ldots \ldots \ldots$ & 14 \\
\hline Gants ou sacs d'introduction diffusants ................... & 7 \\
\hline Gants ou sacs d'introduction désunis de leur support......... & 3 \\
\hline Déchirures ou opérations de déshabillage sur scaphandre...... & 8 \\
\hline 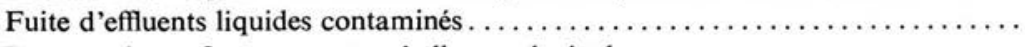 & 13,5 \\
\hline Rupture de confinement sur emballage polyvinyle $\ldots \ldots \ldots \ldots \ldots \ldots \ldots \ldots \ldots \ldots \ldots \ldots \ldots$ & 8 \\
\hline Manipulations sur appareillage pour maintenance $\ldots \ldots \ldots \ldots \ldots \ldots \ldots \ldots \ldots \ldots$ & 5,5 \\
\hline 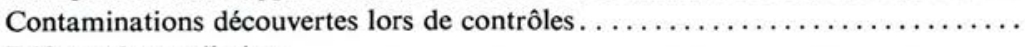 & 6 \\
\hline 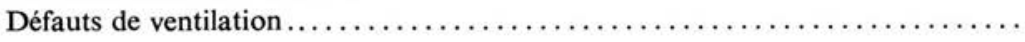 & 3 \\
\hline
\end{tabular}

Ces ruptures de confinement mineures conduisent à des contaminations du milieu, en général faibles, et s'adressent essentiellement aux travailleurs. Des consignes bien comprises, associées à des moyens d'intervention rodés peuvent limiter les conséquences de tels incidents. Si l'on soupçonne une contamination interne, on a la possibilité d'administrer des chélateurs au personnel concerné.

S'il y a des lacunes importantes dans la prévention, des accidents graves peuvent se produire. Le tableau ci-dessous donne quelques exemples d'accidents notables dans lesquels le plutonium était impliqué :

\begin{tabular}{|c|c|c|c|}
\hline Lieu & Année & Type d'accident & Coût \\
\hline $\begin{array}{l}\text { Centre d'études de } \\
\text { Bruyères-le-Châtel } \\
\text { (France) }\end{array}$ & 1962 & $\begin{array}{c}\text { Incendie spontané de Pu }(2 \mathrm{~g}) \\
\text { dans un conteneur : } \\
6850 \text { CMA dans la cellule }\end{array}$ & $\begin{array}{l}6 \text { mois de décontami- } \\
\text { nation pour l'en- } \\
\text { semble du bâtiment } \\
(10 \text { cellules })\end{array}$ \\
\hline $\begin{array}{l}\text { Dow chemical compa- } \\
\text { ny, usine de Rocky } \\
\text { Flats (U.S.A.) }\end{array}$ & 1969 & $\begin{array}{l}\text { Incendie dans un bâtiment : } \\
22000 \mathrm{~m}^{2} \text { contaminés }\end{array}$ & 50 millions de dollars \\
\hline
\end{tabular}

Le tableau IV situe le niveau des risques en cas de dispersion dans le milieu ambiant d'une quantité unitaire de Plutonium, ceci dans l'hypothèse d'un accident détruisant la totalité des barrières. On constate que les valeurs

vol. $11-\mathrm{N}^{0} 3$ 


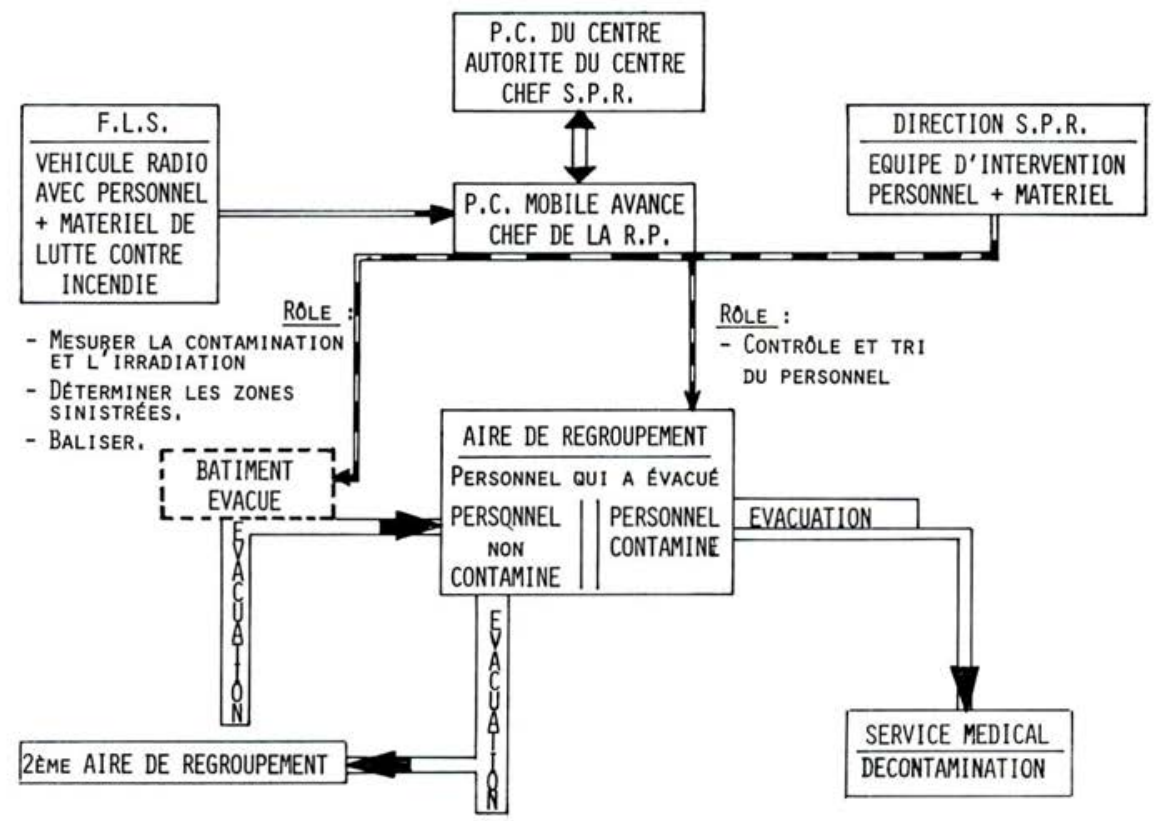

FIg. 16. - Schéma de l'organisation de l'intervention au niveau d'un centre.

TABLEAU IV

Dispersion du Plutonium, estimation des DOSES REÇUeS

\begin{tabular}{|c|c|c|c|}
\hline Distance (en m ) & $\begin{array}{c}\text { Concentration intégrée } \\
\text { pendant le passage } \\
\left.\text { du nuage (Ci.s.m }{ }^{-3}\right)\end{array}$ & $\begin{array}{c}\text { Dépot total au sol } \\
\left(\mu \text { Ci.m }^{-2}\right)\end{array}$ & $\begin{array}{c}\text { Dépot total au sol } \\
\left(\mu \mathrm{g} \cdot \mathrm{m}^{-2}\right)\end{array}$ \\
\hline 500 & $1,7.10^{-3}$ & 17 & 275 \\
1000 & $6,15.10^{-4}$ & 6,15 & 100 \\
5000 & $6,5.10^{-5}$ & 0,65 & 10,5 \\
\hline Distance (en m) & Dose engagée pendant & $\begin{array}{c}\text { Dose engagée par } \\
\text { remise en suspension }(1)\end{array}$ & $\begin{array}{c}\text { Dose engagée par } \\
\text { ingestion }\end{array}$ \\
\hline 500 & 17 rem & 17 rem & 0,17 rem \\
1000 & 6 rem & 6 rem & 0,06 rem \\
5000 & $0,6 \mathrm{rem}$ & $0,6 \mathrm{rem}$ & $0,006 \mathrm{rem}$ \\
\hline
\end{tabular}

Hypothèse : $1 \mathrm{~kg}$ de Pu dispersé; $10 \mathrm{p}$. cent en masse de particules; conditions de diffusion correspondant à $65 \mathrm{p}$. cent des situations météorologiques - légère inversion, vent inférieur à $4 \mathrm{~m} \cdot \mathrm{s}^{-1}$ (facteur 2,7 par rapport aux conditions optimales); vitesse apparente de chute : $10^{-2} \mathrm{~m} \cdot \mathrm{s}^{-1}$.

(1) Dose engagée pour une personne vivant en permanence, sans précautions particulières, sur la zone contaminée correspondante. 
obtenues à partir de bases expérimentales sérieuses, si elles ne sont pas à sous-estimer, sont loin de l'apocalypse décrite par certains.

Comment faire face à ces ruptures de confinements, à ces accidents ? Quels sont les moyens existants, les parades ? D'abord, au niveau des centres nucléaires importants, par l'existence de services spécialisés qui animent et complètent l'action de chacun en matière de sûreté et de sécurité : services de protection contre les rayonnements, services médicaux, services de sécurité incendie, ingénieurs de sécurité du travail.

Ces services, coordonnés, sont chargés de la prévention, d'une grande part de l'action et de la réparation des incidents et des accidents.

En matière de prévention, citons la réalisation d'un plan général en cas de sinistre important (fig. 16).

De tels plans d'intervention ont été généralisés au niveau national regroupant des moyens importants de la Santé Publique, du Service National de la Protection Civile, du Commissariat à l'Énergie Atomique et, selon les circonstances, de l'E.D.F. ou des Armées. Il ne rentre pas dans le cadre de cet article de les décrire, il faut, néanmoins, savoir que l'on procède à des exercices réguliers d'entraînement, malgré la faible probabilité de voir survenir un accident nucléaire.

\section{CONCLUSIONS}

Certains aspects techniques de la radioprotection se posent encore, par exemple :

- Dans quels cas doit-on utiliser une double filtration dans les installations?

- Quelle est l'efficacité des filtres actuels avec des aérosols de sodium-plutonium?

- En cas d'accident doit-on utiliser des chélateurs sans réserve ?

- Comment peut-on améliorer les contrôles de contaminations atmosphériques dans le domaine de l'échantillonnage?

- Doit-on développer la détection par voie aérienne pour des contrôles en cas d'accident ou pour des contrôles périodiques ?

- Comment automatiser sans risque certaines chaînes d'élaboration ? assurée ? etc.

- La sûreté, en cas d'explosion d'enceintes étanches, est-elle bien

Sous un angle plus fondamental, les techniciens qui ont à concevoir des installations, ceux qui se préoccupent plus particulièrement de l'homme au travail, doivent pouvoir s'appuyer sur des bases solides en ce qui concerne la radiotoxicologie et la radiobiologie du plutonium et des transuraniens.

Malgré ces questions qui subsistent, peut-on dire que le plutoinum est dangereux, très dangereux pour l'homme ou, au contraire, qu'il ne pose VOL. $11-\mathrm{N}^{\circ} 3$ 
aucun problème ? La vérité est comme toujours entre ces extrêmes. Si l'on continue à maintenir une prévention importante, lucide, honnête, dans le cadre d'une collaboration étroite de toutes les compétences, on peut affirmer, après 30 ans d'expérience, que le travail sur plutonium est tout à fait possible.

\section{BIBLIOGRAPHIE}

C.E.A. Catalogues Protection, manipulation, détection, sécurité.

C.E.A. Comptes rendus des Groupes de travail du Comité d'instrumentation.

C.E.A. Compte rendu du Groupe de travail Conséquences des accidents (rapport interne).

C.E.A. Guide de manipulation du plutonium et des transuraniens (en cours de révision).

Douellou A. et al. Estimation des débits de dose auprès de différents postes, rapport interne.

Gouguet J. Évaluation des débits d'équivalent de doses dus aux neutrons issus de sources d'oxyde de plutonium. Rapport CEA-R-4666, 1975.

Pages J. P. et Combettes C. Dangers d'irradiation liés à une pièce massive de plutonium fortement irradié. Rapport d'études DAM/B.III/R-69-04-00 0003 DR, 1969.

Risselin A., Chappelier A., Jacquemin M., Bouville A. et Lemenez de Kerdelleau J. Méthode rapide d'estimation des risques. Note CEA (à paraître). 